EPiC Series in Engineering
Volume 3, 2018, Pages 950-958
HIC 2018. 13th International
Conference on Hydroinformatics

\title{
A Real-time Optimal Gate Operation Model for Urban Drainage Systems
}

\author{
Fatemeh Jafari ${ }^{1}$, S. Jamshid Mousavi ${ }^{1}$ and Joong Hoon Kim ${ }^{2}$ \\ ${ }^{1}$ School of Civil and Environmental Engineering, Amirkabir University of Technology (Tehran \\ Polytechnic), Tehran, Iran \\ ${ }^{2}$ School of Civil, Environmental and Architectural Engineering, Korea University, Seoul, South \\ Korea \\ jmosavi@aut.ac.ir
}

\begin{abstract}
Flooding is a phenomenon that endangers human being life and property. There are many structural and non-structural options that can be considered in order to reduce destructive effects of flooding. In this study, we propose a new methodology to enhance the performance of a real-time optimal operation model for flood mitigation in urban drainage systems. An online real-time model is developed as a simulation-optimization approach that leads to optimal operational policies based on the real-time rainfall information. Rainfall-runoff processes and hydraulic routing in the pipes are simulated by the EPA stormwater management model (SWMM) which is linked to the particle swarm optimization (PSO) algorithm, evaluating the system operation performance for assorted sets of operating policies. The initial solution in the real-time model is obtained by a long-term optimal operation model based on historical past flood events. The approach is validated by applying it to a portion of the urban drainage system in Tehran, the capital of Iran, consisting of a prototype network of pipes and detention reservoir equipped with controllable gates. Results show that the proposed strategy in introducing a reasonable initial solution to the real-time model can successfully enhance the performance of the model.
\end{abstract}

Keywords: Urban drainage system, Detention reservoirs, Flood control, Long-term optimization and Real-time operation. 


\section{Introduction}

Urban flooding takes place during severe rainfall events, whenever an urban drainage system (UDS) becomes overloaded. In such a situation, stormwater backs up into the basements and overflows from manholes onto the surface [1]. In order to reduce the flood inundation and related damages, the capacity of the system should be used in the best way; something that can be achieved by proper regulation of the controllable elements in the system such as the detention reservoirs' gates.

Hitherto, various approaches have been applied to adjust gates operation during a storm. One of the traditional methods used is the uncontrolled operation; in which all gates are fully open all the time during flood events. This procedure cannot guarantee the best performance of the system since it does not consider fluctuations of the system loading (incoming flood hydrograph) and dynamic state of the system in policy-making. An alternative approach that overcomes these shortcomings is to make use of an automated control policy by doing optimization. Optimization techniques can lead to better solutions; therefore, they have been widely used in water resources management problems [2], including the operation of gates in UDSs during flood events. Among all different optimization methods, the real-time optimal strategy is one of the most effective approaches to control the operation of a water system, especially in risky flood situations. This is in light of the real-time monitoring of the network and optimal adjustment to different storm events or accidental collapse of a member [3]. Over the last decades, several researchers have investigated the application of real-time control (RTC) techniques to UDS. The first attempt refers to the implementation of an RTC prototype in Minneapolis-St. Paul, United States, at the end of 1960s [4]. Thereafter, numerous assorted RTC strategies have been developed for UDS's operation all over the world. In 2005, a global optimal control system was applied to the Quebec westerly sewer network to minimize set-point variations in real-time and the frequency and volume of sewer overflows discharged into the basin's rivers [5]. Darsono and Labadie (2007) developed an RTC approach based on a neural-optimal algorithm to regulate in-line storages in combined sewer systems [6]. In the combined sewer system of Dresden city, Germany, a global RTC approach was implemented with a hydrodynamic pollution loadcalculation module to improve the efficiency of the sewer system [7]. Garofalo et al (2017) proposed a distributed RTC model using a gossip-based algorithm linked to the SWMM hydrodynamic simulation model [8]. According to the mentioned studies, the dynamic behaviour of RTC approaches leads to a more efficient way of achieving the operational objectives for UDSs. In this research, we enhance the performance of a real-time optimal operation model to regulate gate operation using the results of another optimization technique, i.e. a long-term multi-period optimization approach.

\section{Methodology}

According to Figure (1), suppose a detention reservoir with an outflow gate with a bottom elevation of $\mathrm{B}$ meters above the surface while discretizing the maximum depth of the reservoir ( $H M A X)$ to $\mathrm{n}$ segments. The goal is to develop an optimal operation model to decrease flood inundation at downstream control points of the system. Since the performance of the outflow gate plays a significant role in achieving the goal, i.e. flood reduction, decision variables can be defined as a policy on how to adjust gate openings. Therefore, as shown in Figure (2), the vector of decision variables includes the variables $G_{j}$, each of which represents the percentage of gate opening corresponding to water levels within the $\left[d_{j}, d_{j+1}\right)$ interval that take a value from the set $[0,0.1,0.2$, $\ldots, 1]$. 
Obviously, the gate starts working as the water level in the detention reservoir exceeds the bottom elevation of the gate, i.e. height $B$ in Figure 1. Thus, the number of decision variables depends on this height. For the aforementioned example, if the maximum height is divided into 10 segments $(n=10)$, the number of decision variables is equal to $m=7$. Adding more gates to the system will increase the number of decision variables.

Different operation models can be used in order to determine an operation policy (percentage of gate opening conditioned on water level) and evacuate water out of the system. We propose a realtime optimal operation model that is then enhanced based on the results obtained by applying a longterm optimal operation model.

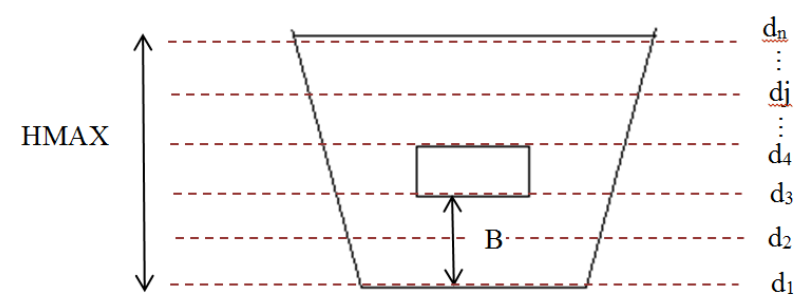

Figure 1: Discrete water level in the detention reservoir

\begin{tabular}{|l|l|l|l|l|l|l|}
\hline$G_{1}$ & $G_{2}$ & $G_{3}$ & $\ldots$ & $G_{j}$ & $\ldots$ & $G_{m}$ \\
\hline
\end{tabular}

Figure 2: Decision variables vector

\subsection{Long-term optimal (LTOP) operation model}

In this offline model, a set of historical storm events are used as the input to the system to derive a specific optimal operation policy. The derived policy determines an optimal gate opening corresponding to each discrete water level in the detention reservoir. The policy can then be applied to control future flood events. Therefore, the regulation of the gates is performed based on the water level as the state of the system [9]. Such a unique rule of this method may not be the best one, but it can be considered as a good solution for the initial state of another optimization approach.

\subsection{Real-time optimal (RTOP) operation model}

In this approach, the time horizon of operation $(D)$ is divided into a number of decision times $T_{i}$ for which a specific control rule $R_{i}$ is determined by analyzing available real-time input data. Consequently, a finite sequence of operating policies $R_{1}, R_{2}, \ldots, R_{i}, \ldots, R_{H}$ is produced over the time horizon $D$. Each $R_{i}$ includes a vector of optimal policies for gate operation (as shown in Figure (2)), which is employed only during the period of decision time $T_{i}$ and would be updated for the next period.

It can be inferred that in this model, not only the state of the system impacts the system operation, but also the attributes of each particular flood event is taken into account for policy-making. This is an advantage in comparison to other models such as LTOP model. Despite all positive aspects of this approach, its performance can still be improved. One way to improve the efficiency and performance 
of the RTOP model is to use the derived rule by the LTOP model as the initial solution for the RTOP model. Figure (3) presents the scheme of how these two optimization approaches are linked together. The LTOP operation model is executed using a set of several historical storm events to produce the long-term optimal operation rule, which is then imported as the initial solution of the optimization module in the RTOP model. The particle swarm optimization (PSO) algorithm is used to find optimal policies at each decision time and is linked to the SWMM simulation model, evaluating the objective function value.

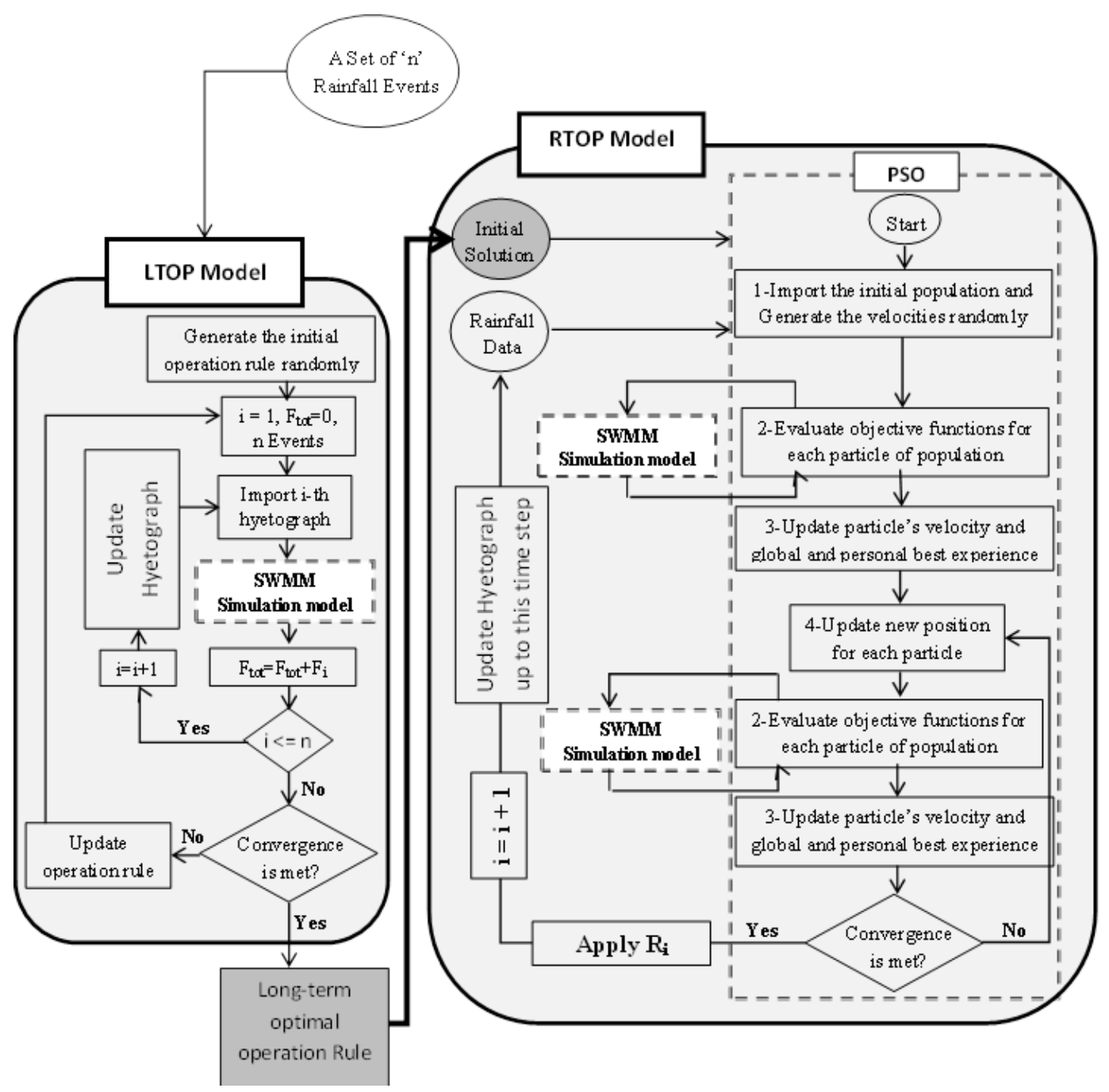

Figure 3: Flowchart of the proposed model 


\section{Case Study}

A part of the main drainage system located in Tehran, the capital of Iran, is used to investigate the efficiency of the proposed model through several severe rainfall events. The drainage network consists of $116 \mathrm{~km}$ underground tunnels of which approximately $15.6 \mathrm{~km}$ does not have an adequate capacity to safely transfer stormwater runoff. Figure (4) illustrates the location of the detention reservoir and it's concrete outlet intake structure with three steel sluice gates each having $1.6 \times 1.6 \mathrm{~m}^{2}$ size, eight rectangular openings of $0.6 \times 0.9 \mathrm{~m}^{2}$ size and a three diameter octagonal opening on its roof. The characteristics of the detention reservoir are displayed in Table (1). Table (2) presents the characteristics of utilized events. A total number of 25 rainfall events with different characteristics occurred between 1974 to 2013 are selected to derive the optimal long-term operation rule of the LTOP model, and six intense rainfall events are used to validate the performance of the model.

\begin{tabular}{cl}
\hline Maximum Depth $(\mathrm{m})$ & \multicolumn{1}{c}{7.5} \\
\hline & EL $0: 85000$ \\
Area $\left(\mathrm{m}^{2}\right)$ & EL 1: 160000 \\
& EL 7.5: 160000 \\
\hline
\end{tabular}

Table 1: Detention reservoir characteristics

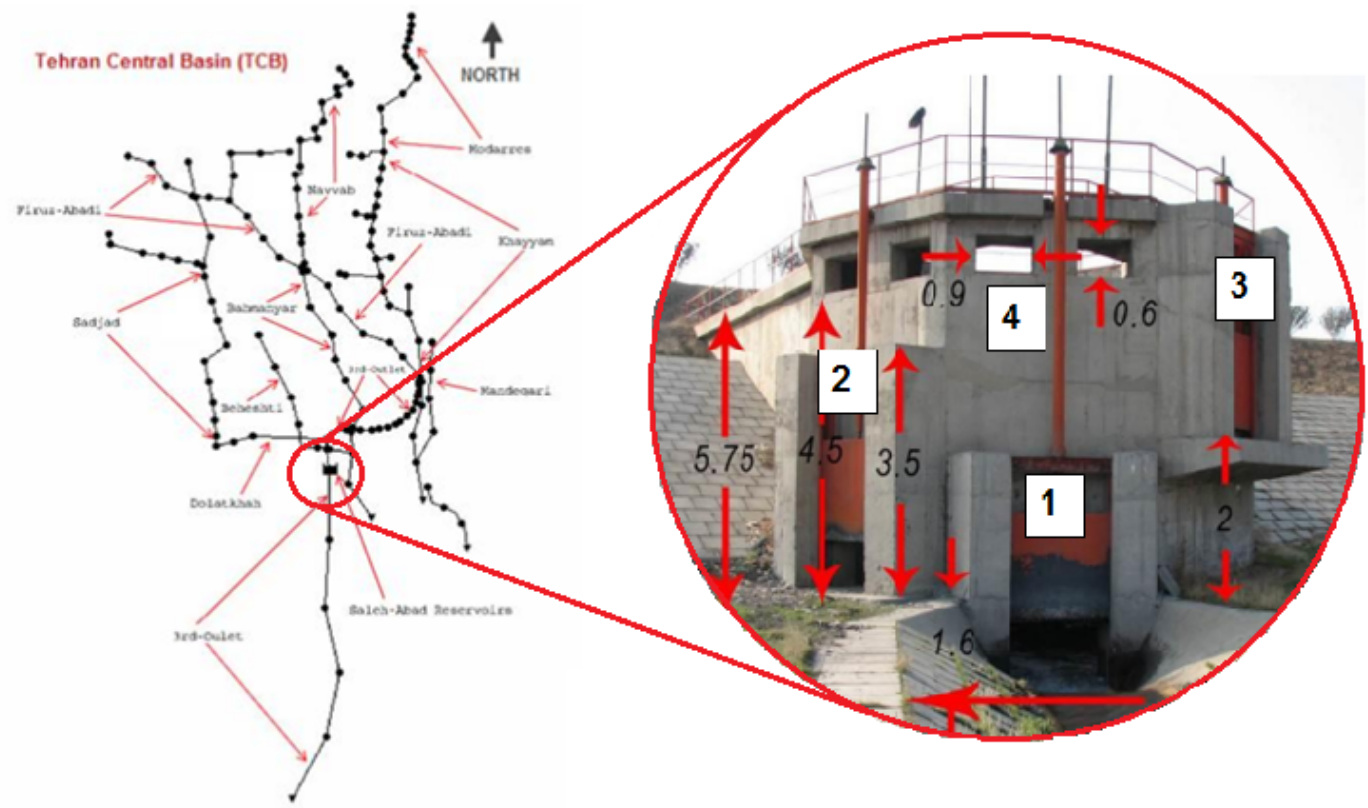

Figure 4: Schematic representation of the studied network and outlet intake structure of the detention reservoir

\section{Results and Discussion}

Figure (5) illustrates the SWMM model of the investigated UDS that is developed to simulate the performance of the system. System's data, features, and characteristics were collected by MG 
Consulting Engineers (MGCE) [10]. The network was divided into two sub-models as shown in Figure (5) in order to decrease the computational load by eliminating the repetitive calculations. So, for each decision time, the upstream sub-model is called just for one time and the downstream submodel is run for each function evaluation. This procedure is used based on the assumption that gravity-flow is established in the upstream model that is independent of gate performance. The assumption was validated by comparing the results obtained for integrated (Figure (4)) and separated (Figure (5-a)) networks for different events.

The allowable maximum water depth in the detention reservoir was equal to HMAX $=7.5 \mathrm{~m}$ and was divided into 15 discrete values with 0.5 -meter increments. The decision variable vector was defined as the percentages of openings corresponding to each discrete water level for four gates of the reservoir (Figure (4)). Therefore, 38 decision variables were considered to optimize the operation of gates. The variables are 15,12,8 and 3 variables related to the sluice gates 1, 2, 3 and all openings together (4), respectively.

The PSO algorithm was utilized to determine the optimal operation policy that was improved using an adaptive inertia weight [11]. The PSO model parameters were fine-tuned by trial runs for several events (see Table 5). Using an Intel Core i7 $3.2 \mathrm{GHz}$ system with $16 \mathrm{~GB}$ of RAM, the proposed simulation-optimization algorithm takes 13 minutes to converge and create optimal policies for each 30-minute decision time period.

Figure (6) presents the long-term rule derived from the LTOP model using twenty-five extreme rainfall events. An operation policy has been determined for all eight rectangular gates (Gate 4 in Figure (4)) as they are quite similar and located at the same elevation.

Applying the derived rules as the initial solution for the RTOP model and performing several computer experiments, we observed that the proposed model successfully reduce flood inundation in comparison with the traditional approach, i.e. uncontrolled operation method that is currently in practice (Figure (7)). Additionally, using the proposed strategy, the convergence speed of the RTOP model has improved significantly, and the computational load required to reach the best solution has considerably decreased. Table (4) shows the impact of model improvement in terms of flood reduction.

\begin{tabular}{|c|c|c|c|c|c|c|c|}
\hline No. & Event & $\begin{array}{l}\text { Duration } \\
\text { (min) }\end{array}$ & $\begin{array}{c}\text { Accumulation } \\
\text { of } \\
\text { precipitation }(\mathrm{mm})\end{array}$ & No. & Event & $\begin{array}{l}\text { Duration } \\
\text { (min) }\end{array}$ & $\begin{array}{c}\text { Accumulation } \\
\text { of } \\
\text { precipitation }(\mathrm{mm})\end{array}$ \\
\hline \multicolumn{4}{|c|}{ Six Validating storm Events } & \multicolumn{4}{|c|}{ Rainfall events using in LTOP model } \\
\hline 1 & 29-Dec-76 & 610 & 21.22 & 10 & $05-F e b-89$ & 785 & 28.37 \\
\hline 2 & 26-Jan-80 & 235 & 15.4 & 11 & 09-May-89 & 535 & 17.89 \\
\hline 3 & 07-Dec-84 & 230 & 25.71 & 12 & 25-Mar-90 & 265 & 20.29 \\
\hline 4 & 28-Mar-02 & 190 & 9.14 & 13 & 04-Apr-91 & 600 & 25.18 \\
\hline 5 & 04-May-04 & 405 & 8.75 & 14 & 03-Mar-93 & 775 & 32.4 \\
\hline 6 & 15-Jul-12 & 270 & 28.7 & 15 & 26-Oct-94 & 30 & 10.49 \\
\hline \multicolumn{4}{|c|}{ Rainfall events using in LTOP model } & 16 & 02-Mar-96 & 1380 & 45.81 \\
\hline 1 & 15-Jan-74 & 1650 & 29.72 & 17 & 29-Mar-97 & 825 & 21.23 \\
\hline 2 & 20-Feb-75 & 1595 & 45.15 & 18 & 03-Dec-98 & 1145 & 39.45 \\
\hline 3 & 25-Apr-77 & 505 & 12.63 & 19 & 12-Nov-99 & 1380 & 31.75 \\
\hline 4 & 20-Feb-81 & 685 & 16.35 & 20 & 30-Dec-00 & 890 & 34.23 \\
\hline 5 & 27-Mar-81 & 605 & 13.15 & 21 & $13-F e b-02$ & 880 & 27.29 \\
\hline 6 & 14-Nov-82 & 440 & 16.13 & 22 & 17-May-03 & 945 & 39.61 \\
\hline 7 & 16-Mar-84 & 370 & 14.16 & 23 & 31-Jan-11 & 265 & 10.3 \\
\hline 8 & 18-Dec-85 & 1480 & 39.67 & 24 & 28 -Oct-11 & 1325 & 61.5 \\
\hline 9 & 03-May-86 & 1680 & 38.32 & 25 & 14-Apr-13 & 185 & 18.8 \\
\hline
\end{tabular}

Table 2: Detention reservoir characteristics 


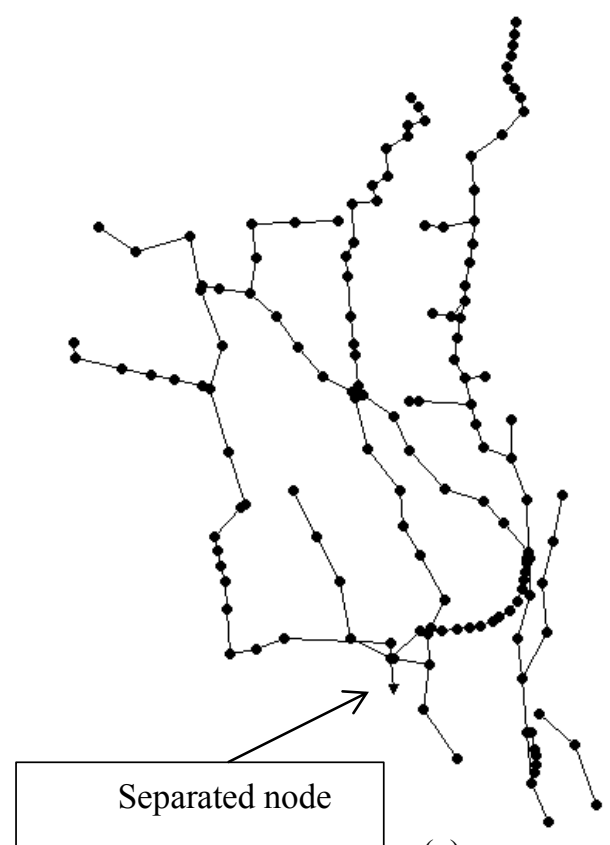

(a)

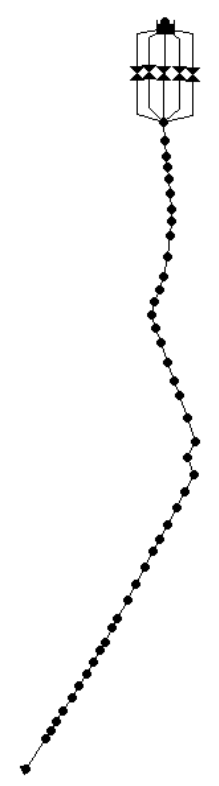

(b)

Figure 5: Simulation sub-models (a) upstream sub-model, (b) downstream sub-model
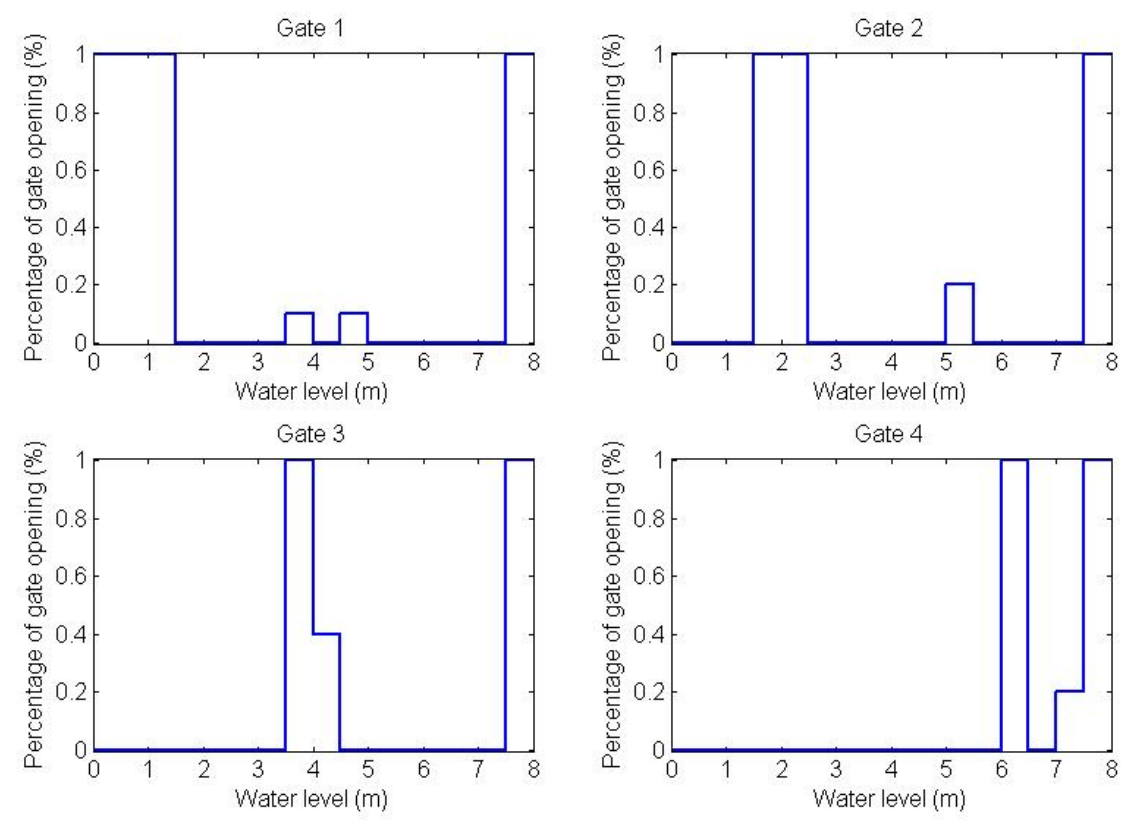

Figure 6: Long-term optimal operation rule obtained by the LTOP model 
According to the Table (4), improving the RTOP model using a reasonable initial solution has led to a reduction of flood inundation up to $17 \%$.
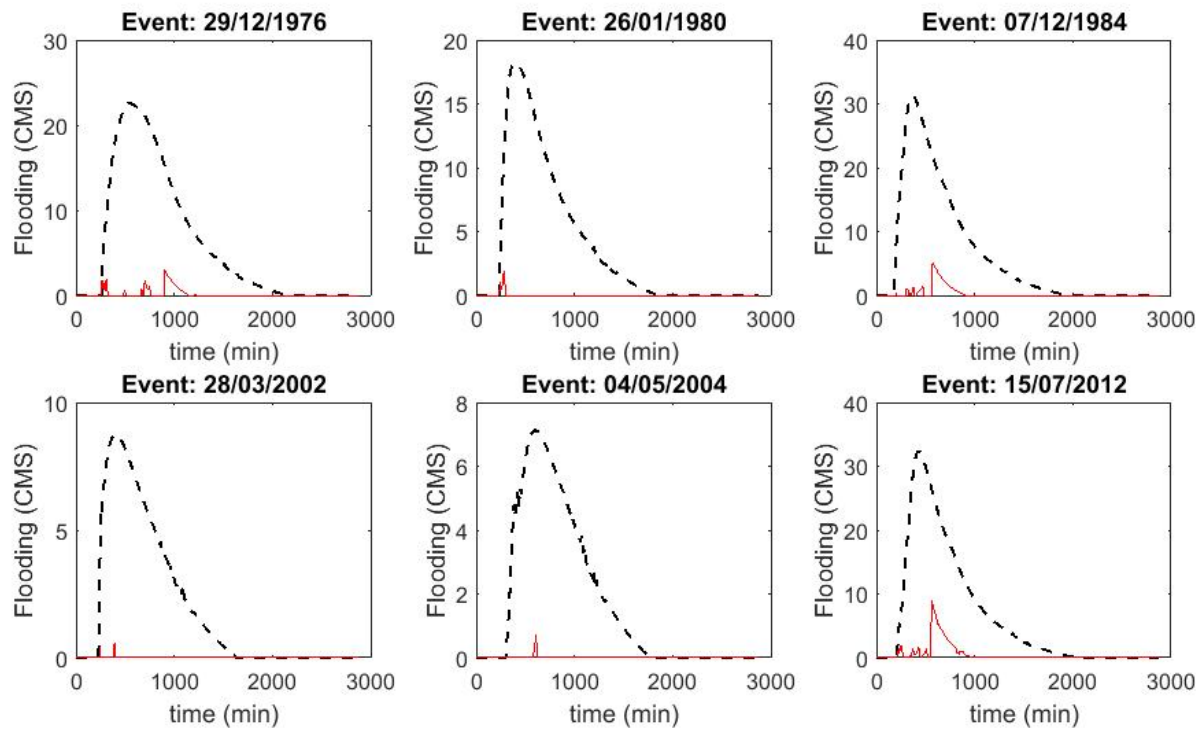

Uncontrolled Operation

Real-time Optimal Operation

Figure 7: of uncontrolled operation model and RTOP operation model in terms of flood inundation

Table 3: Parameters used in the PSO algorithm

\begin{tabular}{|c|c|c|}
\hline \multicolumn{2}{|c|}{ Parameters } & Value \\
\hline \multicolumn{2}{|c|}{ Number of population } & 100 \\
\hline \multirow{2}{*}{ Inertia weight $(\mathrm{w})$} & $\mathrm{W}_{\min }$ & 0.8 \\
\hline & $\mathrm{W}_{\max }$ & 1.2 \\
\hline \multicolumn{2}{|c|}{ Personal learning constant $\left(\mathrm{C}_{1}\right)$} & 2 \\
\hline \multicolumn{2}{|c|}{ Global learning constant $\left(\mathrm{C}_{2}\right)$} & 2 \\
\hline \multicolumn{2}{|c|}{ Maximum velocity $\left(\mathrm{V}_{\max }\right)$} & +0.8 \\
\hline \multicolumn{2}{|c|}{ Minimum velocity $\left(\mathrm{V}_{\min }\right)$} & -0.8 \\
\hline \multicolumn{2}{|c|}{ Maximum Iteration } & 100 \\
\hline
\end{tabular}

\begin{tabular}{cccc}
\hline & \multicolumn{2}{c}{ Flooding (1000 m3) } & Flood Reduction (\%) \\
\cline { 2 - 3 } Event & $\begin{array}{c}\text { RTOP Operation } \\
\text { model }\end{array}$ & $\begin{array}{c}\text { Improved RTOP Operation } \\
\text { model }\end{array}$ & \\
\hline 29-Dec-76 & 34.55 & 28.51 & 17 \\
26-Jan-80 & 0 & 0.30 & - \\
07-Dec-84 & 45 & 44.42 & 1 \\
28-Mar-02 & 0 & 0.07 & -- \\
04-May-04 & 0 & 0.10 & - \\
15-Jul-12 & 86.93 & 83.78 & 4 \\
\hline
\end{tabular}

Table 4: Impact of proposed improvement in terms of flood reduction 


\section{Conclusion}

One of the efficient solution approaches to manage flooding in urban areas is the real-time control approach. Adding an optimization module to this approach would be an effective means to take the full advantage of a UDS capacity. In this study, a real-time optimal operation model was proposed in order to mitigate flood inundation in an urban drainage system located in the Tehran, the capital of Iran. The PSO optimization algorithm was worked collaboratively with SWMM simulation model to generate real-time optimal operation policies for the operation of the detention reservoir gates of the system. To enhance the model performance, a long-term optimal operation rule, which was derived using a long-term optimal operation model, was used as the initial solution of the optimization module. The results demonstrated that the proposed framework could significantly decrease the flooding volume in comparison with an uncontrolled operation approach that has been in practice in the region. Additionally, the strategy of using a rational initial solution led to the reduction of the computational load that is essential to the success of real-time control approaches.

\section{References}

[1] Garofalo G, Giordano A, Piro P, Spezzano G, Vinci A, A distributed real-time approach for mitigating CSO and flooding in urban drainage systems. Journal of Network and Computer Applications. 2017 Jan 15;78:30-42.

[2] Hsu NS, Huang CL, Wei CC. Intelligent real-time operation of a pumping station for an urban drainage system. Journal of hydrology. 2013 May 10;489:85-97.

[3] Dirckx G, Schütze M, Kroll S, Thoeye C, De Gueldre G, Van De Steene B. RTC versus static solutions to mitigate CSO's impact. In12nd International Conference on Urban Drainage, 2011b. Porto Alegre, Brazil 2011 Sep.

[4] Borsanyi, P., Benedetti, L., Dirckx, G., De Keyser, W., Muschalla, D., Solvi, A. M., ... \& Vanrolleghem, P. A. (2008). Modelling real-time control options on virtual sewer systems. Journal of Environmental Engineering and Science, 7(4), 395-410.

[5] Pleau M, Colas H, Lavallée P, Pelletier G, Bonin R (2005) Global optimal real-time control of the Quebec urban drainage system. Environmental Modelling \& Software, 20(4), 401-413.

[6] Darsono S, Labadie JW (2007) Neural-optimal control algorithm for real-time regulation of inline storage in combined sewer systems. Environmental Modelling \& Software, 22(9), 1349-1361.

[7] Beeneken, T., Erbe, V., Messmer, A., Reder, C., Rohlfing, R., Scheer, M., ... \& Weyand, M. (2013). Real time control (rtc) of urban drainage systems-a discussion of the additional efforts compared to conventionally operated systems. Urban Water Journal, 10(5), 293-299

[8] Garofalo, G., Giordano, A., Piro, P., Spezzano, G., \& Vinci, A. (2017). A distributed real-time approach for mitigating CSO and flooding in urban drainage systems. Journal of Network and Computer Applications, 78, 30-42.

[9] Jafari, F., Mousavi, S.J., Yazdi, J. and Kim, J.H.. Long-term versus real-time optimal operation for gate regulation during flood in urban drainage systems. Submitted to the Urban Water Journal.

[10] MGCE (2011a) Tehran Stormwater Management Master Plan, Vol 4: Existing Main Drainage Network, Part 2: Hydraulic Modeling and Capacity Assessment, December 2011, MG Consultant Engineers, Technical and development deputy of Tehran municipality, Tehran, Iran.

[11] Nickabadi, A., Ebadzadeh, M. M., \& Safabakhsh, R. (2011). A novel particle swarm optimization algorithm with adaptive inertia weight. Applied Soft Computing, 11(4), 3658-3670. 